\title{
Fine-Needle Aspiration Biopsy of Metastatic Malignant Melanoma Resembling a Malignant Peripheral Nerve Sheath Tumor
}

\author{
Svetoslav Bardarov, M.D., Claire W. Michael, M.D., \\ David Lucas, M.D., Yijun Pang, M.D., Ph.D., \\ and Robert T. Pu, M.D., Ph.D.
}

\begin{abstract}
We report a case of metastatic malignant melanoma resembling a malignant peripheral sheath tumor, which posed a significant diagnostic challenge. The patient is a 76-year-old male, who presented in the emergency room with bilateral chest pain exacerbated by inspiration. The pain was present for 3 week and was not exacerbated by physical exercise. The diagnostic workup revealed bilateral parenchymal pulmonary infiltrates. The CT-scan guided fine-needle aspiration and the core biopsies of the largest pulmonary lesion revealed high-grade spindle cell neoplasm with individual cell apoptosis and necrosis. The immunohistochemical profile on the cell block showed that the cells are positive for Vimentin. The S-100 stain showed only focal positivity. The immunohistochemical stains for HMB45, Melan A, pancytokeratin, and smooth muscle actin were negative. Five years ago the patient was diagnosed with melanoma on the back with Clark level of IV. The melanoma was excised with clear margins and sentinel lymph nodes were negative. Careful examination of patient's previous slides revealed an area of spindle cell melanoma adjacent to a nodular type melanoma. Based on the patient's previous history, current clinico-pathologic presentation and immunohistochemical profile, the diagnosis of metastatic malignant melanoma resembling peripheral nerve sheath tumor was favored over the diagnosis of metastatic malignant spindle cell neoplasm of unknown primary site, which by itself is very rare clinical scenario. Diagn. Cytopathol. 2008;36:754-757. @ 2008 Wiley-Liss, Inc.
\end{abstract}

Key Words: melanoma; MPNST; immunohistochemistry

\footnotetext{
Department of Pathology, University of Michigan, Ann Arbor, Michigan

*Correspondence to: Claire W. Michael, M.D., Department of Pathology, 1500 East Medical Drive, Ann Arbor, MI 48109.

E-mail: clairemi@med.umich.edu

Received 26 March 2008; Accepted 16 May 2008

DOI 10.1002/dc.20893

Published online in Wiley InterScience (www.interscience.wiley.com).
}

Melanoma is an increasingly common, potentially fatal form of skin cancer arising from the malignant transformation of melanocytes. Currently, melanoma is the seventh most common type of cancer and the most common malignancy in women ages $25-29 .{ }^{1}$ Melanoma has the ability to spread and give metastasis to different sites sometimes years after the initial diagnosis is made. Primary as well as metastatic melanomas can have different morphologic features on light microscopy, which make the diagnosis difficult. It can resemble small round cell tumor, poorly differentiated carcinomas as well as low and high-grade soft tissue sarcoma. Because of the morphologic heterogeneity, malignant melanoma is always in the differential diagnosis of metastatic tumors of unknown primary site. In this report, we present a case of metastatic melanoma which morphologically resembles malignant peripheral nerve sheath tumor, which posed a significant diagnostic challenge.

\section{Case Reports}

A 76-year-old man presented to the emergency room complaining of sharp bilateral chest pain associated with shortness of breath. The pain worsened with coughing and inspiration. The pain had been present for the prior 3 weeks, was stable and was not exacerbated with walking or physical activity. The patient's past medical history included: hypertension, atrial fibrillation, and melanoma on the back excised about 5 years ago. The patient's social and family history was noncontributory. Chest Xray revealed large pleural effusion on the left. Computer tomography scan revealed multiple bilateral opacities, the 

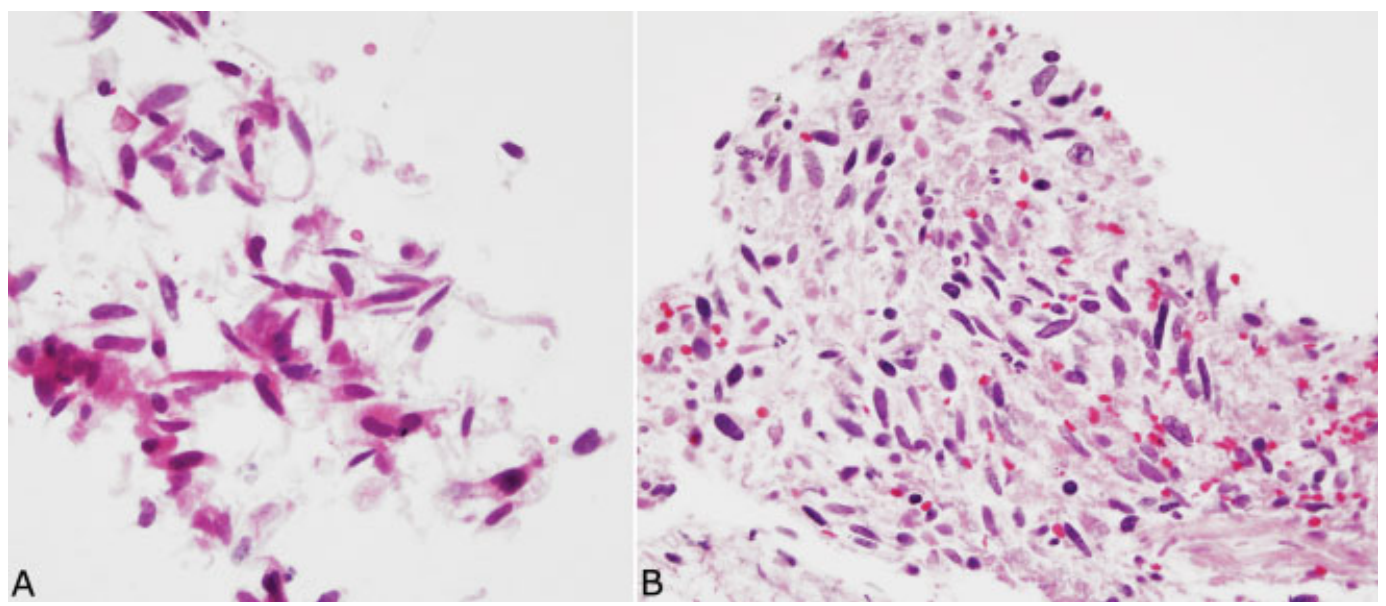

Fig. 1. Fine-needle aspiration biopsy of patients current disease. (A) Cell block $(H \& E, \times 200)$ and $($ B) Core biopsy obtained during the same procedure $(\mathrm{H} \& \mathrm{E}, \times 200)$. [Color figure can be viewed in the online issue, which is available at www.interscience.wiley.com.]

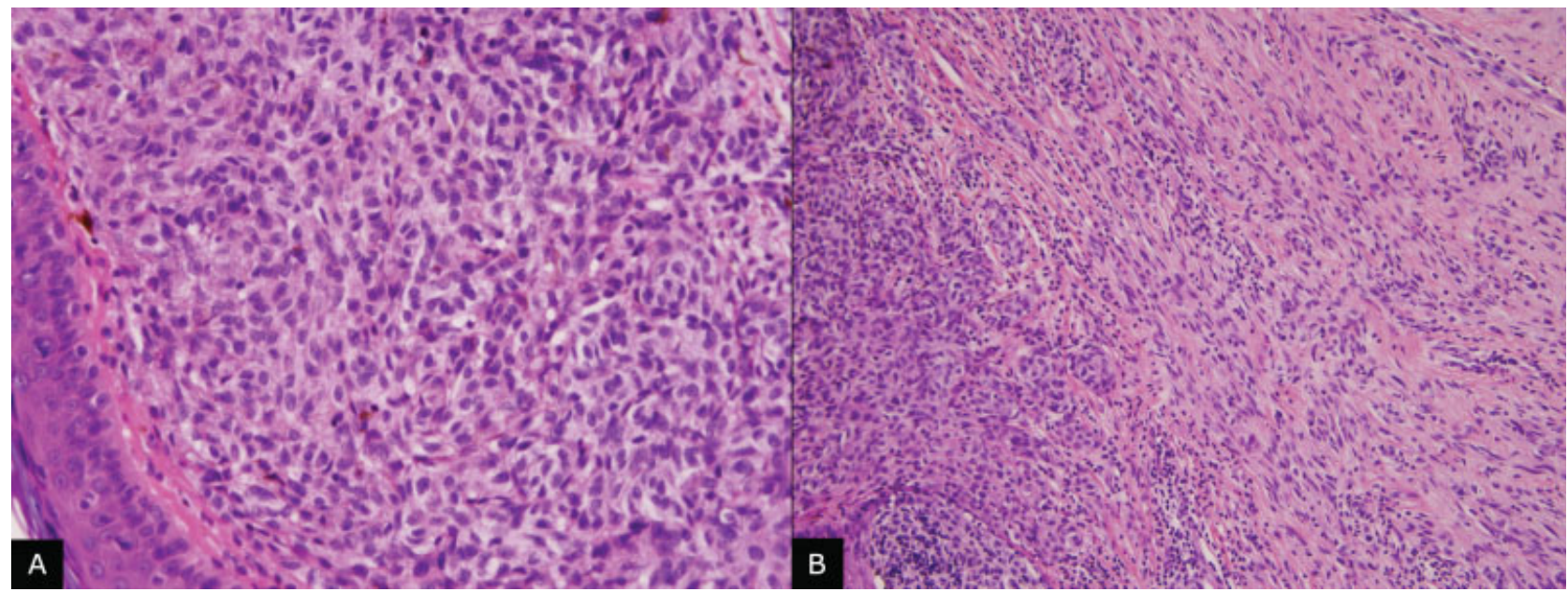

Fig. 2. Examination of the patients previously diagnosed melanoma revealed: (A) Usual type nodular melanoma and (B) Areas adjacent to the nodular melanoma showing spindle cell melanoma but no necrosis (H\&E, $\times 200)$. [Color figure can be viewed in the online issue, which is available at www. interscience.wiley.com.]

largest one measuring $3.5 \mathrm{~cm}$ on the left lower lobe. The patient was referred for CT-scan guided fine-needle aspiration and core biopsy of the largest pulmonary lesion.

\section{Cytological Finding}

The examination of the cell block revealed a paucicellular specimen composed of scattered fragments of spindled cells, scattered leukocytes, and individually apoptotic malignant cells (Fig. 1A). Occasional larger, epithelioid cells containing a moderate amount of cytoplasm, slightly enlarged hyperchromatic nuclei containing small "cherry red" nucleoli were also noted (Fig. 1B). One important morphologic sign one should keep in mind is that the nucleoli of the metastatic melanomas are usually oxyphilic, whereas the macro nuclei of the malignant peripheral nerve sheath tumor are usually basophilic. The tissue core biopsies obtained during the same procedure showed similar appearing cells with large areas of necrosis, cellular pleomorphism, and cellular apoptosis. The tumor cells were mainly discohesive, however, clumps of malignant looking cells with necrosis and acute inflammation were also noted.

\section{Past Medical History and Pathology}

Diagnostic slides from the patient's previously diagnosed melanoma (2004) were obtained, which showed both invasive and in-situ Malignant Melanoma with Clark level IV (Breslow depth of $1.2 \mathrm{~mm}$ ) (Fig. 2). The margins of excision were free of tumor. A total of nine sentinel lymph nodes were obtained and showed no evidence of metastatic melanoma. The original case showed nodular type of melanoma composed of pigment containing plasmacytoid cells with abundant cytoplasm and single 


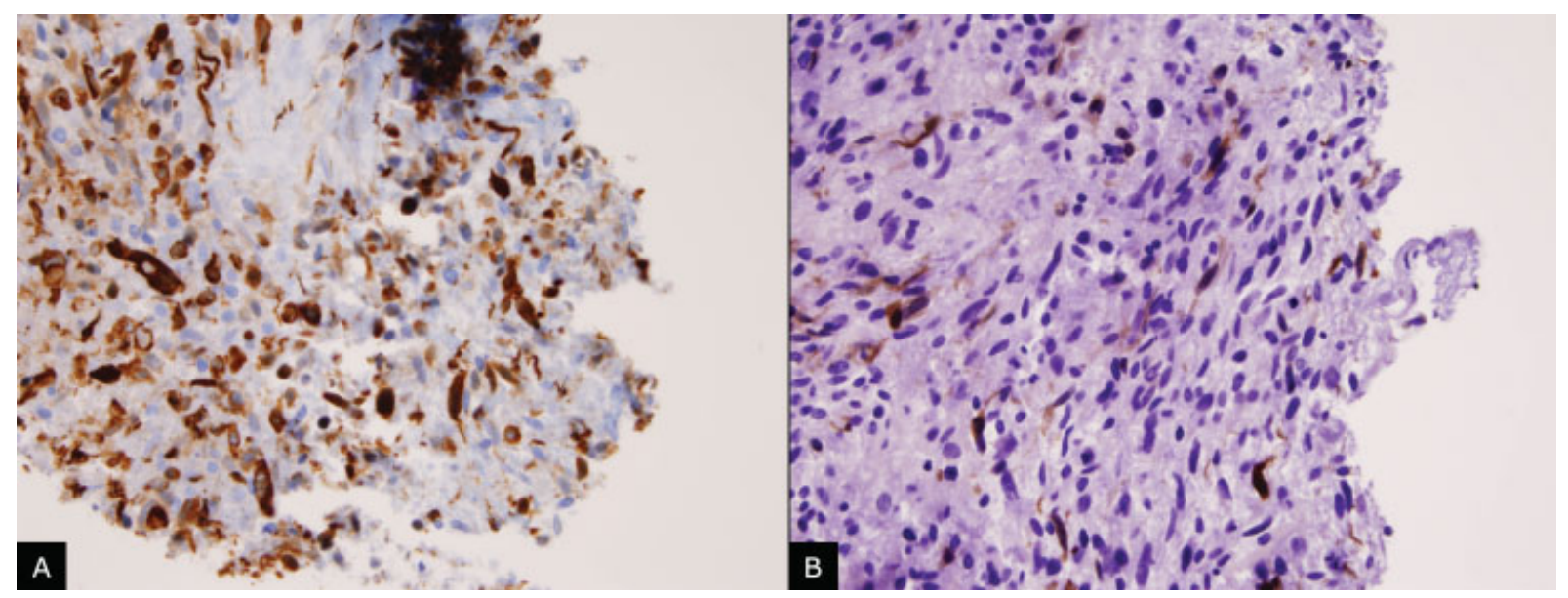

Fig. 3. Immunohistochemical profile of the patient current disease. (A) Vimentin was positive in many cells and (B) S-100 shows only rare positive cells $(\mathrm{H} \& \mathrm{E}, \times 200)$. [Color figure can be viewed in the online issue, which is available at www.interscience.wiley.com.]

"cherry red" nucleoli. Adjacent to the usual type nodular melanoma, there was an area with spindle cells with nuclear atypia and scant melanin pigment (Fig. 2B). No necrosis or mitoses were seen on the original slides.

\section{Immunohistochemistry}

The immunohistochemistry were performed on paraffin sections of the core biopsies as well as on the cell block, with the use of avidin-biotin peroxidase complex technique. The tumor cells were negative for Melan A, HMB45, Calretinin, CK7, CK20, CD15, TTF-1, and Pancytokeratin. Additional stains for smooth muscle actin were negative as well. The tumor cells showed strong positivity for Vimentin (Fig. 3) and focal positivity for S100 (Fig. 3B).

\section{Discussion}

This is a case with unusual histological features and immunohistochemical profile (focal positivity for S100 only), which created a significant diagnostic dilemma. Morphologic comparison of the original tumor and current malignancy reveal similar area of spindle cell melanoma adjacent to the nodular type of melanoma. The differential diagnosis included metastatic spindle cell melanoma, metastatic malignant peripheral nerve sheath tumor, and sarcomatoid mesothelioma.

The incidence of melanoma had increased world wide. The most recent analysis of global cancer statistics, from 2002, demonstrated and incidence of 6.4 cases per 100,000 men and 11.7 cases per 100,000 women in North America. ${ }^{2}$ This malignancy is known to have many faces and can present with variable morphology. It can present with epithelioid, signet ring, balloon, and plasmacytoid cells and in some cases it can resemble malignant highgrade sarcoma, including malignant peripheral nerve sheath tumor. ${ }^{3-5}$ In one study King et al. reviewed and described in detail the overlapping features of metastatic melanomas and malignant peripheral nerve sheath tumors. ${ }^{4}$ The authors presented 16 metastatic tumors all of which were positive for Vimentin and showed variable positivity for S100 and HMB45. Only one case showed focal staining with S100 similar to the case we describe. The remaining cases showed strong immunoreactivity for S100 and 5 out of 16 cases were positive for HMB45. In a similar study, Piao et al. retrospectively reviewed 81 metastatic spindle cell melanomas diagnosed by fine-needle aspiration. ${ }^{6}$ Those authors observed that $20 \%$ of the metastatic spindle cell melanomas had dissimilar morphology to their primary counterparts. In the same study $72 \%$ of the tumors showed positivity for at least one melanoma marker. The distribution of the S100, HMB45, and MelanA was $67 \%, 50 \%$, and $31 \%$, respectively. Aisner et al. $^{7}$ observed that $1 \%$ of metastatic melanomas S100 immunoreactivity was lost. The authors studied $1553 \mathrm{mel}-$ anomas, $17(1 \%)$ of which were negative for S100. Of those $59 \%$ were immunoreactive for Mart-1 or GP100 and 13 had documented primary melanoma. ${ }^{7}$

Malignant peripheral nerve sheath tumor is a rare neoplasm which occurs in conjunction with neurofibromatosis type $1 .^{8}$ The life-time risk of developing MPNST in the general population was estimated to be $0.001 \%$, however this increases up to as high as $10 \%$ in patients with preexisting neurofibromatosis. ${ }^{8}$ The 5 year survival of MPNST is $\sim 64 \%$. These tumors very rarely can contain melanin and when this pigment is observed the differential diagnosis from metastatic malignant spindle cell melanoma is rather challenging. Tumor size, grade as well as presence or absences of distant metastasis are factors of crucial prognostic importance. Similar to stage I and stage II melanoma, small volume low-grade tumors have a good prognosis, in contrast to less than $50 \% 3$ year survival when metastatic disease is present.

The cytomorphologic features of malignant peripheral nerve sheath tumor have been reviewed by Klijanienko 
Table I. Cytomorphologic Features of Metastatic Spindle Cell Melanoma Resembling MPNST vs. MPNST

\begin{tabular}{|c|c|c|c|}
\hline & Features & Spindle cell melanoma resembling MPNST & MPNST \\
\hline \multirow[t]{2}{*}{ Clinical Presentations } & Prerequisites & History of melanoma & $\begin{array}{l}\text { Neurofibromatosis type } 1 \\
\text { History of MPNST }\end{array}$ \\
\hline & Presentation & $\begin{array}{l}\text { Can represent with metastatic } \\
\text { disease of unknown primary site }\end{array}$ & $\begin{array}{l}\text { Metastatic disease as initial } \\
\text { presentation is very rare }\end{array}$ \\
\hline \multirow[t]{3}{*}{ Low-power features } & Cellularity & Variable & Variable \\
\hline & Background & Can have large areas of necrosis & Fibrilary or Myxoid \\
\hline & Cellular arrangement & Mostly discohesive & $\begin{array}{l}\text { Clusters; Fascicular and } \\
\text { parallel arrangement }\end{array}$ \\
\hline \multirow[t]{8}{*}{ High-power features } & Cellular Morphology & $\begin{array}{l}\text { Spindle cells with sharp ends; } \\
\text { occasional epithelioid } \\
\text { cells present }\end{array}$ & $\begin{array}{l}\text { Fusiform nuclei } \\
\text { with round ends }\end{array}$ \\
\hline & $\mathrm{N}: \mathrm{C}$ ratio & Increased & Variable \\
\hline & Pleomorphism & Variable & Variable \\
\hline & Cytoplasmic vacuoles & None & Variable \\
\hline & Nucleoli & Oxyphillic & Basophilic \\
\hline & Mitotic figures & Many & Variable \\
\hline & Apoptosis & Present & None \\
\hline & Intranuclear inclusions & Present & Rare \\
\hline
\end{tabular}

et al. $^{9}$ and described in great detail by Gupta et al. ${ }^{10}$ In summary MPNST can present with variable cellularity on FNA, usually with clusters of cells arranged in fascicular or parallel pattern. The nuclear pleomorphism is variable with variable mitotic activity. Nucleoli are usually present and basophilic and the cytoplasm may contain vacuoles. Nuclear inclusions usually are not present and individual cell apoptosis is rarely observed.

King et al. ${ }^{4}$ advanced a list of clinicopathological features that may serve as guidelines for the diagnosis of $\mathrm{MM}$ mimicking MPNST. Briefly, the diagnosis of metastatic MM should be entertained when one or more of the following are seen in a spindle cell neoplasm: (1) tumor localized within or close to a lymphnode; (2) absence of continuity with a major nerve or a neurofibroma, or absence of neurofibromatosis; (3) presence, or history of past primary MM; (4) strong and diffuse S-100 protein immunoreactivity (S100 protein is usually focal and/or weak in MPNSTs); and (5) immunoreactivity for other melanoma markers. It is possible that in the clinical practice one encounter a patient with history of melanoma and MPNST and in these cases the differentiation of between both neoplasms may be impossible. The cytomorphologic features which may assist in this difficult cases are: presence of fibrilary or myxoid background, fascicular arrangement, basophilic nucleoli, cytoplasmic vacuoles, and no nuclear inclusions all of which will favor MPNST over metastatic spindle cell malignant melanoma (Table I).

In this article, we presented a case which posed a significant diagnostic challenge. The patient presented with a metastatic high-grade spindle cell neoplasm, with prominent necrosis. He had a previous history of melanoma with Clark lever IV, however, with negative sentinel nodes. The patient had no history of neurofibromatosis and no other known primary malignancies were detected. The lesion was negative for melanoma markers and showed only focal positivity for S-100 which favored a MPNST. Based on the clinical history of malignant melanoma as well as identification of similar appearing spindle cells in the original slides, we favor the diagnosis of metastatic malignant melanoma over metastatic high-grade spindle cell neoplasm of unknown primary site. Even though the presence of high-grade bulky metastatic disease confers a poor prognosis for this patient it is important to discriminate between both neoplasms in order to guide future treatments such as experimental treatment plans or protocols.

\section{References}

1. Rigel DS, Friedman RJ, Kopf AW. The incidence of malignant melanoma in the United States: Issues as we approach the 21 st century. J Am Acad Dermatol 1996;34:839-847.

2. Parkin DM, Bray F, Ferlay J, Pisani P. Global cancer statistics, 2002. CA Cancer J Clin 2005;55:74-108.

3. Lodding P, Kindblom LG, Angervall L. Metastases of malignant melanoma simulating soft tissue sarcoma. A clinico-pathological, light- and electron microscopic and immunohistochemical study of 21 cases. Virchows Arch A Pathol Anat Histopathol 1990;417:377388.

4. King R, Busam K, Rosai J. Metastatic malignant melanoma resembling malignant peripheral nerve sheath tumor: Report of 16 cases. Am J Surg Pathol 1999;23:1499-1505.

5. Banerjee SS, Harris M. Morphological and immunophenotypic variations in malignant melanoma. Histopathology 2000;36:387402.

6. Piao Y, Guo M, Gong Y. Diagnostic challenges of metastatic spindle cell melanoma on fine-needle aspiration specimens. Cancer 2008;114(2): 94-101.

7. Aisner DL, Maker A, Rosenberg SA, Berman DM. Loss of S100 antigenicity in metastatic melanoma. Hum Pathol 2005;36:1016-1019.

8. Ferner RE, Gutmann DH. International consensus statement on malignant peripheral nerve sheath tumors in neurofibromatosis. Cancer Res 2002;62:1573-1577.

9. Klijanienko J, Caillaud JM, Lagace R, Vielh P. Cytohistologic correlations of 24 malignant peripheral nerve sheath tumor (MPNST) in 17 patients: The Institut Curie experience. Diagn Cytopathol 2002;27:103-108.

10. Gupta K, Dey P, Vashisht R. Fine-needle aspiration cytology of malignant peripheral nerve sheath tumors. Diagn Cytopathol 2004; 31:1-4. 